\title{
Multicast Routing Protocol with Heterogeneous and Dynamic Receivers*
}

\author{
Huimei Lu, Hongyu Hu, Quanshuang Xiang, and Yuanda Cao \\ School of Computer Science and Technology, \\ Beijing Institute of Technology, Postcode 1000 81, \\ 5\# Zhongguancun south road, Haidian district, Beijing, P.R. China \\ \{Blueboo, ydcao\}@bit.edu.cn, \\ $\{$ hu_hong_yu, xiangquanshuang\}@163.com
}

\begin{abstract}
This paper aims to design an effective multicast routing supporting heterogeneous and dynamic receivers. Multicast data is assumed to be layered cumulatively. The multicast tree constructed fulfills the QoS requirements imposed by heterogeneous receivers in terms of the layer of the data and its corresponding bandwidth, and consumes network resource as little as possible. Besides the general state information of the topology and the available bandwidth, two kinds of group-specific state information such as the distribution of multicast tree and the highest receivable layer of on-tree nodes are maintained at a router to calculate a feasible graft path to the multicast tree. Simulation results demonstrate that the proposed protocol obtains low routing message overhead, high success routing ratio and optimization usage of network bandwidth.
\end{abstract}

\section{Introduction}

Supporting heterogeneous receivers in a multicast session is particularly important in large networks as, e.g., the Internet, due to the large diversity of end-system and network access capabilities [1]. To accommodate heterogeneous receivers, multicast data can be encoded into a base layer and several successive enhancement layers, and then receivers in a multicast session can receive different layers according to their bandwidth capabilities. One approach to realize this is that the source sends all the layers of a session on a single IP multicast group along and the involved routers decide which layers to forward. This is called network level schemes [2][3] which aim to find a multicast tree that can support heterogeneous QoS requirements of the receivers.

Research works that support heterogeneous multicast with dynamic receivers can be found in Refs.[2][4]. The problem in these researches is that when the receiver computes the feasible path, it excludes paths without enough residual bandwidth from consideration even though part of the path lies on the multicast tree and bandwidth has already been reserved. Thus, if the quality level of the new receiver is higher than that of on-tree nodes, it maybe fails to find an existing feasible path. QDMR-LD [5] is

\footnotetext{
* This work was supported by the national Natural Science Foundation of China under contract number 60503050 and University Basic Foundation of Beijing Institute of Technology under contract number BIT UBF 200511F4212.
} 
designed to support dynamic receivers. When a new receiver wants to join, it floods join-request messages towards the multicast tree. The join-request message is forwarded based on RBMF mode [6]. QDMR-LD has high success routing ratio and minimal bandwidth usage. But, it is a fully distributed algorithm and is based on flooding, which causes excessive routing message overhead.

Most of the previous researches are based on the general network information such as, topology and link states. Through careful observation, we find some group-specific information plays great role in an effective routing algorithm.

This paper proposes a new multicast routing for dynamic and heterogeneous receivers (MRDH), which is designed to inherit the merits of QDMR-LD and solve its disadvantage. Besides the general state information of the topology and the available bandwidth, two kinds of group-specific state information such as the distribution of multicast tree and the highest receivable layer of on-tree nodes are selected to be maintained in this study. Based on the above state information, a routing algorithm is designed which calculate a feasible tree branch locally and effectively.

The rest of the paper is organized as follows. Section 2 presents the network model, the data model and the routing formulation. Section 3 gives a detailed description of the protocol. Simulation results are demonstrated in Section 4. Section 5 draws our conclusions.

\section{System Model}

\subsection{Network Model}

The network is modeled as a digraph $N=(R, S N, C)$, where $R$ is the set of routers, $S N$ is the set of all physical subnets and $C$ is the set of joints between routers and physical subnets. The bandwidth available on subnet $s n_{i}$ is denoted as $b\left(s n_{i}\right)$. The network topology structure is described by the graph constructed by cycles, rectangles and edges which represent subnet, router and NIC on the router respectively, as shown in Fig. 1.

A multicast tree $T$ is a sub-graph of $N$, which can be represented as $T=\left(R_{T}, S N_{T}\right)$ $\left(R_{T} \subseteq R, S N_{T} \subseteq S N\right)$, where $R_{T}$ is the set of routers, including the relay routers on the multicast tree, and $S N_{T}$ is the set of the subnets connecting the routers belong to $R_{T}$. In Fig. 1, for example, a source lies in subnet $s n_{1}$, and two receivers lie in subnet $s n_{5}$ and $s n_{10}$ respectively. The tree covers routers of $R_{1}, R_{2}, R_{5}$, and subnets of $s n_{3}, s n_{7}$.

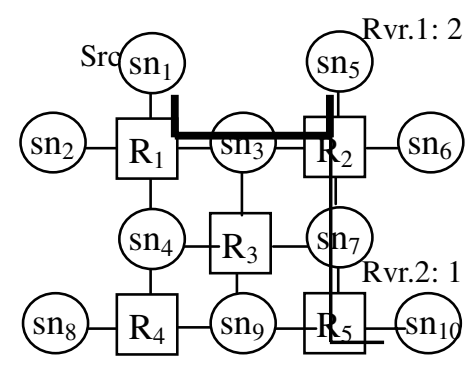

Fig. 1. Network model 


\subsection{Traffic Model for Layered Data}

There are two kinds of layering schemes: cumulative and non-cumulative [1]. In this paper, we suppose traffic is encoded cumulatively. The signal is encoded hierarchically into $L$ layers with the first layer containing the most essential information, and information in Layer $i$ is less significant than those in Layer $i-1$, but more significant than those in Layer $i+1$. Thus, a signal with higher layers has better quality and at the same time requires more bandwidth for transmission. Only layers that a given subnet can manage are forwarded. In Fig. 1, suppose the source encoded the data into 2 layers. Receiver 1 has high access capability, and its request is 2 layers. Receiver 2 is a mobile phone, and its request is 1 layer.

\subsection{Routing Formulation}

For any on-tree router $R_{u}\left(R_{u} \in R_{T}\right)$, it stores a multicast routing entry, composed by the multicast source, the group address, the upstream router and the set of downstream routers and their respective layers. The quality level of router $R_{u}$, denoted as $l_{u}$, is represented by the max layer among all the layers of downstream routers.

Considering $R_{u}$ receives a join-request with $l$ layers, if $l>l_{u}$, it can not decide whether it can satisfy the request independently. However, if it keeps the highest receivable layer $M R_{u}$, it can make a decision by itself. The value of $M R_{u}$ is decided by the highest receivable layer of the upstream router $R_{t}$ and the available bandwidth of the upstream subnet $s n$. Its calculation method refers to (1).

$$
M R_{u}=\min \left(M R_{t}, f\left(f^{1}\left(l_{u}\right)+b(s n)\right)\right) .
$$

Where, function $f$ defines the relationship between the data layer and the corresponding bandwidth. For example, In Fig. $1, M R_{2}=2$. For router $R_{5}$, if the available bandwidth on subnet $s n_{7}$ is high enough, its highest receivable layer is 2 .The highest receivable layer of a multi-access subnet is represented by the max value of that of down-stream routers.

Formulation. When a new receiver $d$ intends to join a group with $k$ layers, it sends the join-request to its designated router $R_{d}$. $R_{d}$ will try to find a path from any on-tree nodes $u$ to itself, denoted as $P\left(u, R_{d}\right)$, which satisfies the following constraints:

$$
\begin{gathered}
M R_{u} \geq k . \\
b\left(P_{i}\left(u, R_{d}\right)\right)=\min \left\{b(s n) \mid\left(s n \in P_{i}\left(u, R_{d}\right)\right) \&\left(s n \notin S N_{T}\right)\right\} \geq f^{1}(k) . \\
h\left(P\left(u, R_{d}\right)\right)=\min \left\{h\left(P_{i}\left(u, R_{d}\right)\right) \mid i=0, . . \mathrm{N}\right\} . h(P) \text { means the hops on path } P
\end{gathered}
$$

\section{Proposed Protocol}

In this section, we describe our protocol in detail, especially the join situation where a new receiver wants to join a multicast group.

When a receiver wants to join a multicast group, it issues a join-request message with $k$ layer and forwards the message to its Designated Router. DR calculates a feasible path $P\left(u, R_{d}\right)$ locally. Fig. 2 shows the detailed calculation procedure. 


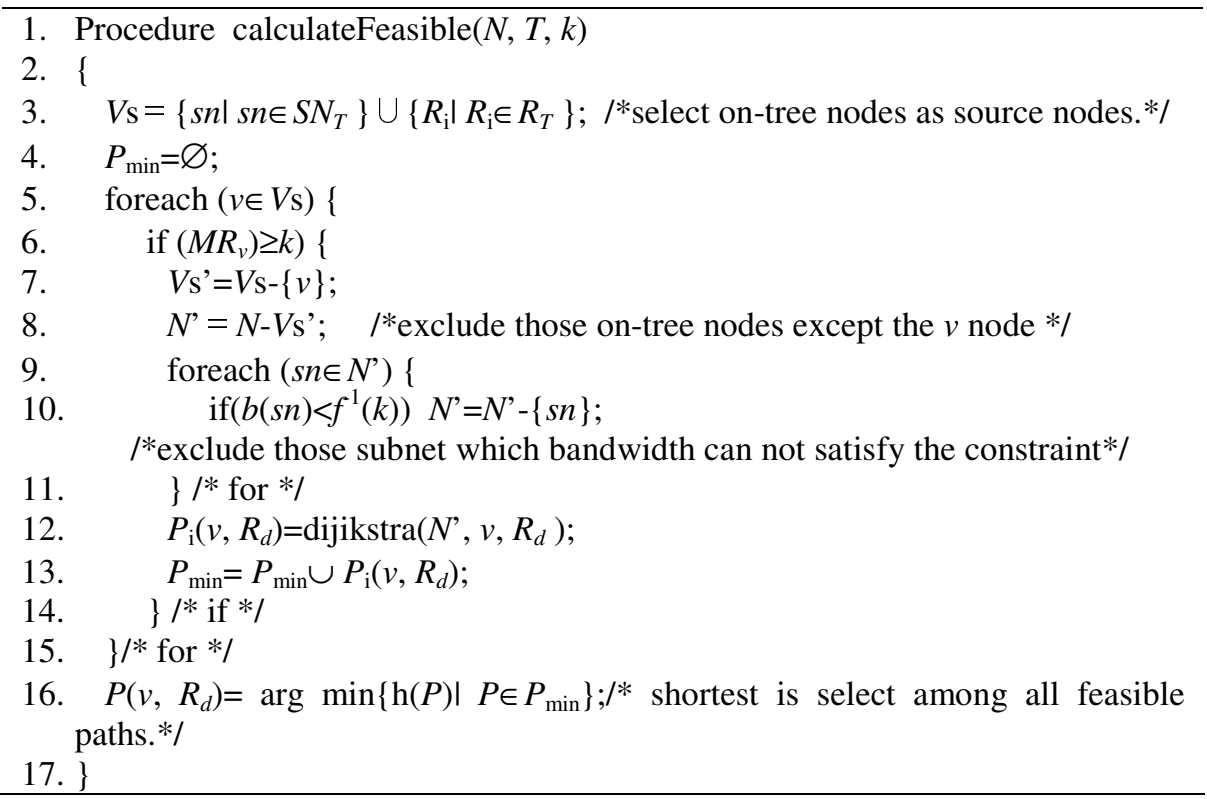

Fig. 2. The algorithm for calculation of a feasible graft path

If the feasible graft path exists, DR sends a join-request message along the path to inform associated routers to set up connection; Otherwise DR refuses the receiver's join request.

When an off-tree router receives the join-request message, it simply sends the message according to the path information stored in the message. When an on-tree node receives the message, it first checks whether its current highest receivable layer is higher or equal to the new receiver's join request layer. If it is not, it replies DR a retry message to re-calculate again to find another feasible path. Else, it then checks whether its current layer is higher or equal to the request layer. If it is not, it will continue to forward the join-request to the upstream router; else it will do the followings: (1) Update the multicast routing entry by adding the new downstream routers and its receivable layer; (2) Reserve corresponding bandwidth caused by the additional layer; (3) Reply the set-up message to the new downstream router. When the set-up message arrives at DR finally, the new receiver has been connected to the multicast tree. It should be noted that the network information should be updated and broadcasted in the range of the whole network wide because both the bandwidth on the graft path and the distribution of the multicast tree are changed.

\section{Simulation Results}

The simulation study is conducted on the Network Simulator (NS-2) [8] platform and on the topology of CERNET [9]. MRDH is compared with two dynamic algorithms, QDMR-LD[5], QMRP-LD [7] and one static algorithm Maxenchuk [3] in three performance metrics which are defined as follows: 
Avg. msg. overhead= total number of Join-request msg. sent / total number of join members

Success ratio $=$ number of new receivers accepted $/$ total number of join members Bandwidth used per member $=$ total bandwidth used by successful receivers $/$ number of new successful receivers.

In our experiment, the bandwidth of each group is uniformly distributed from 2 to 6 Mbps. Video data is encoded into three layers: a base layer, a most significant layer, and an enhancement layer with the bit rates being $30 \%, 50 \%$, and $20 \%$ of the total video respectively. 500 groups are launched gradually in this experiment. The source and the members of each group are selected randomly, however only the simulation results of the middle join rate (10 nodes among 36 nodes join to each group as the member) is shown for the length limitation of this paper. The receiving capability of each receiver is randomly selected among 1, 2, 3 layers.

Fig. 3 gives the simulation results of the three performance metrics. MRDH computes a feasible graft path locally and then sends a join-request message along the path to set up the branch. QMRP-LD unicasts a join-request message from the DR towards the core. If a router in the unicast path does not satisfy the QoS requirement, the join-request message backtracks to the former router and then is flooded to all neighbor routers. As shown in Fig. 3(a), the message overhead of MRDH is much lower than that of QDMR-LD, and almost as low as that of QMRP-LD when the group number is small; but becomes much lower than that of QMRP-LD when group number increases. In Fig. 3(b), with the increase of group number, the percentage of receivers accepted decreases more significantly under QMRP-LD, while decreases gently under MRDH, QDMR-LD and Maxenchuk's algorithm. QMRP-LD relies on unicast routing to find a feasible path without considering the network bandwidth already reserved for a multicast session. Therefore, the success ratio under QMRP-LD is lower than that of MRDH. In Fig. 3(c), the multicast tree constructed under MRDH is more efficient in resource usage compared to that under QDMR-LD and QMRP-LD, and close to that under Maxenchuk's algorithm for one of the most important aims of Maxenchuk's algorithm is to minimize the total bandwidth resources used.

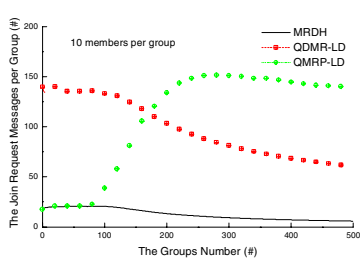

(a)

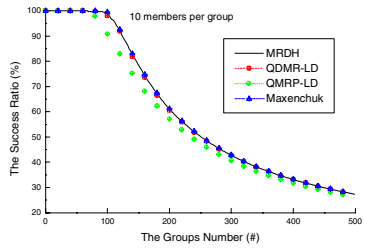

(b)

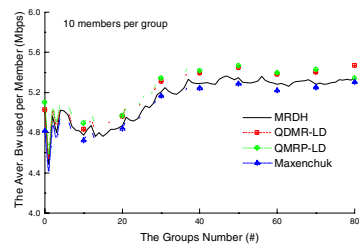

(c)

Fig. 3. Performance comparison of (a) the message overhead; (b) the success routing ratio; (3) the average bandwidth used per member under different algorithms

In conclusion, MRDH has the following prominent advantages: (1) MRDH can calculate in a centralized way by storing two kinds of group-specific information. Feasible paths are calculated locally by DRs, the message overhead for searching 
feasible paths tends to be very low. (2) If the network state information is up-to-date, the success ratio tends to be high. Unlike others routing, MRDH does not rely on any unicast routing. Hence, every router knows the bandwidth reserved for existing multicast group, which improves the probability of finding an existing feasible path. (3) The network bandwidth usage is optimized. The graft path is the shortest path among all the feasible paths. It leads to efficient use of network resources.

Certainly, MRDH has to maintain a global state, which limits its usage in large networks. But the above pros confirm that MRDH is an effective multicast routing in small range of networks.

\section{Conclusions}

In this paper, we study the problem of constructing a multicast tree for heterogeneous and dynamic receivers. We demonstrate that by maintaining the distribution of multicast tree and the highest receivable layer of on-tree nodes, feasible paths can be calculated locally. We then describe our protocol in a detailed way. Simulation results shows that our routing tends to have low message overhead, high routing success ratio and optimization usage of network bandwidth.

\section{References}

1. Li, B., Liu, J.C.: Multirate Video Multicast over the Internet: An Overview. IEEE Network. Volume 17.(2003)2-6

2. Lui, K.-S., Wang, J., Xiao, L., Nahrstedt K.: QoS Multicast Routing with Heterogeneous Receivers. In: Proceedings of IEEE GLOBECOM. (2003) 3597-3601

3. Maxemchuk, N. F.: Video Distribution on Multicast Networks. IEEE Journal on Selected Areas in Communications. Volume 15. (1997) 357-372

4. Wang, B., Hou, J. C.: QoS-Based Multicast Routing for Distributing Layered Video to Heterogeneous Receivers in Rate-based Networks. In: Proceedings of IEEE INFOCOM. (2000) 480-489

5. Lu, H.M., Xiang Y., Shi M.L., Yang M.: A QoS-Based Dynamic Multicast Routing Algorithm for Streaming Layered Data. Journal of Software. Volume 15. (2004) 928 939

6. Lu, H.M., Xiang Y., Shi M.L., Yang M.: A New Bandwidth and Delay-Constrained Distributed Multicast Routing. ACTA ELECTRONICA SINICA. Volume 20. (2002) 1978-1981

7. Chen, S., Nahrstedt, K., Shavitt, Y.: A QoS-Aware Multicast Routing Protocol. In: Proceedings of IEEE INFOCOM. (2000) 1594-1603

8. Network Simulator ns-2.available from http://www.isi.edu/nsnam/

9. CERNET. Available from http://www.edu.cn/HomePage/cernet_fu_wu/about_cernet/ 